\title{
STEM CELLS FROM HUMAN EXFOLIATED DECIDUOUS TEETH - ISOLATION, LONG TERM CULTIVATION AND PHENOTYPICAL ANALYSIS
}

\author{
Jakub Suchánek1, Benjamín Vǐšek ${ }^{2}$,Tomáš Soukup ${ }^{2}$, Sally Kamal El-Din Mohamed ${ }^{3}$, Romana Ivančaková1, \\ Jaroslav Mokry $\tilde{y}^{2}$ Eman H.A. Aboul-Ezz ${ }^{3}$, Omran A. ${ }^{4}$
}

Charles University in Prague, Faculty of Medicine and University Hospital Hradec Králové, Czech Republic: Department of Dentistry ${ }^{1}$, Department of Histology and Embryology2; Oro-Dental Genetic Department, National Research Center, Cairo, Egypt ${ }^{3}$; Faculty of Dentistry, Ein-Shams University, Cairo, Egypt ${ }^{4}$

\begin{abstract}
Summary: Aims: Our aims were to isolate stem cells from human exfoliated deciduous teeth (SHED), to cultivate them in vitro and to investigate their basic biological properties, phenotype and to compare our findings with dental pulp stem cells (DPSC) isolated from permanent teeth. Methods: Dental pulp was gently evacuated from exfoliated teeth. After enzymatic dissociation of dental pulp, SHED were cultivated in modified cultivation media for mesenchymal adult progenitor cells containing 2 \% FCS and supplemented with growth factors and insulin, transferrin, sodium (ITS) supplement. Cell viability and other biological properties were examined using a Vi-Cell analyzer and a Z2-Counter. DNA analyses and phenotyping were performed with flow cytometry. Results: We were able to cultivate SHED over 45 population doublings. Our results showed that SHED cultivated under same conditions as DPSC had longer average population doubling time (41.3 hrs for SHED vs. $24.5 \mathrm{hrs}$ for DPSC). Phenotypic comparison of cultivated SHED to that of cultivated DPSC showed differential expression CD29, CD44, CD71, CD117, CD166. During long-term cultivation, SHED did not showed any signs of degeneration or spontaneous differentiation. Conclusions: We isolated stem cells from exfoliated teeth. In comparison to DPSC, SHED proliferation rate was about $50 \%$ slower, and SHED showed slightly different phenotype. These cells may be extremely useful for stem cell tissue banking, further stem cell research and future therapeutic applications.
\end{abstract}

Key words: Dental pulp; Stem cells; Exfoliated deciduous teeth; Isolation; Cultivation; Phenotype

\section{Introduction}

Stem cells can be divided broadly into two categories embryonic and adult. Embryonic stem cells are pluripotent, capable of differentiation into virtually any mature cell type. These cells can be propagated indefinitely in an undifferentiated state.

Due to ethical issues associated with the use of embryonic stem cells, recent attention has focused on stem cells derived from adult tissues. It is thought that adult stem cells have a more restricted differentiation potential compared to embryonic stem cells. Nevertheless adult stem cells still fulfill the basic characteristics of stem cells - abilities to self-renew, generate large numbers of progeny and differentiate into multiple mature cell types. The ability of these cells to regenerate tissues of all germ layers leads to the thought that they may find great use in stem cell regenerative therapy of tissues damaged by disease, aging or trauma.
Mesenchymal stem cells (MSC) are adult multipotent stem cells that have two unique properties. The first one is their capacity of self renewal beyond Hayflick's limit, a property shared by embryonic stem cells. The second is the ability of MSC to differentiate into mesenchymal and nonmesenchymal mature cell lines such as fat, bone, cartilage (6) and neural cells (7). Until recently, postnatal stem cells have been isolated almost from all adult tissues (bone marrow, neural tissue, skin, retina, etc.) (10). Findings that MSC can be relatively easily isolated from various tissues and subsequently expanded render them a promising tool for regenerative medicine. These stem cells are thought to possess great therapeutic potential for repairing damaged tissues.

Multipotent MSC with the ability to undergo osteogenic and chondrogenic differentiation as well as to regenerate tooth-specific structures such as cementum have been isolated from the dental pulp $(3,4)$. In these experiments dental pulp tissue was isolated from impacted $3^{\text {rd }}$ molars. In 
2003 Miura et al (5) isolated stem cells from human exfoliated deciduous teeth (SHED). In their experiments, after transplantation- the cells were able to form significant amount of bone in vivo after transplantation. Dental pulp from exfoliated teeth was concluded to be an alternative source of postnatal stem cells (5).

It has been demonstrated that dental pulp stem cells (DPSC) are able to regenerate a dentine-pulp-like complex that is composed of mineralized matrix with odontoblasts and fibrous tissue in an arrangement similar to the dentine - pulp complex found in normal human teeth $(2,12)$. Tooth regeneration is one of the ultimate goals of restoring the loss of natural teeth. Some studies have indicated that cell-based strategies show promising potential for regenerating the whole tooth structure in rodents $(1,11)$. Moreover, stem cell-based regeneration of human tooth structures has been achieved in imunocompromised mouse models (3).

The transition from deciduous teeth to adult permanent teeth is a very unique and dynamic process. The development and eruption of permanent teeth is coordinated with the resorption of the roots of deciduous teeth. This process starts at about 6 years and stops after 12 years of age. In this time period all of the 20 deciduous teeth are normally replaced.

Dental pulp is well defined compartment of soft tissue which retains a primitive structure similar to that of the gelatinous tissue of the umbilical cord. Because of the specific "SHED niche", we proposed that these cells would share characteristics of primitive stem cells. Thus the possibility to isolate a population of multipotent stem cells from the remaining pulp of exfoliated deciduous teeth could offer a unique stem-cell resource for potential clinical applications when extraction of these teeth would cause any harm in future life. We undertook this study to investigate this possibility and compare the cells derived from culture of SHED to that from culture of DPSC under conditions designed to enhance stem cell proliferation and differentiation.

\section{Materials and Methods}

Teeth were obtained from 3 children undergoing deciduous tooth extraction aged from 7-9 years (shedding period) and from 5 adult donors undergoing third molars extraction (18-20 years old). Legitimate representatives of all patients provided informed consent according to guidelines of the Ethical Committee of the Faculty Hospital in Hradec Králové.

Collection and transport of extracted teeth: The extraction procedure was done under standard conditions in local anesthesia. Extracted teeth were treated by disinfection solution and - together with the pulp - transported in Hanks' balanced salted solution (HBSS, Invitrogen, USA) to the tissue cultures laboratory.

Isolation, digestion and cultivation: Dental pulp (DP) was isolated under sterile conditions. Isolation of DP from third molars was harder, because those teeth had fully developed roots. We separated them from the crown using Luer's forceps. In deciduous teeth the roots were partially resorbed and dental pulp chamber was widely opened (Fig. 1). We used sharp needle or excavators to release DP from the pulp chamber, after wide access was granted. Extracted dental pulp from deciduous and from adult teeth was then enzymatically treated with collagenase type I ( $3 \mathrm{mg} / \mathrm{ml}$, Sevapharma, CR) and dispase ( $4 \mathrm{mg} / \mathrm{ml}$, Invitrogen, USA) for 35 minutes to completely digest the pulp tissue. Following centrifugation at $600 \mathrm{~g}$ (2000 rpm for 5 minutes), the cell pellet was obtained and the supernatant aspirated.

DPSC and SHED were cultivated in $5 \% \mathrm{CO}_{2}$ atmosphere under $37^{\circ} \mathrm{C}$ in a previously described media (9) composed of $\alpha$-MEM (Invitrogen, USA), 2 \% FCS (PAA, Austria), 10 ng/ml EGF (Peprotech, USA), 10ng/ml PDGF (Peprotech, USA), L-ascorbic acid (Sigma, USA), glutamine (Invitrogen, USA), penicilin/streptomycin (Invitrogen, USA), gentamycin (Invitrogen, USA), dexamethasone (Sigma, USA) and supplemented with $10 \mu \mathrm{l} / \mathrm{ml}$ ITS (Sigma, USA). SHED were cultivated for 3-5 days in primary culture in culture flasks with "Cell ${ }^{+}$" surface (Sarstedt, USA), then treated with trypsin-EDTA (Invitrogen, USA) and split into culture flasks with standard tissue cultures - treated surfaces (TPP, Switzerland or NUNC, USA). Each following passage was carried out after reaching $70 \%$ confluence.

Cell analysis: Viability was assessed using ViCell analyzer (Beckman Coulter, USA) based on trypan dye exclusion method. Z2 counter (Beckman Coulter, USA) measurement is based on Coulter's principle. For flow cytometry analyses, cells were detached and stained sequentially with immunofluorescent primary antibodies (FITC- or PE-labeled) until analysis with a Cell Lab Quanta (Beckman Coulter, USA) flow cytometer. DNA analysis was performed by DNA Prep kit (Beckman Coulter, USA) based on propidium iodide staining, according to manufacturer's instructions.

The percentage of positive cells was determined, as percentage of cells with higher fluorescence intensity than the $99.5 \%$ of negative isotype control. Classification criteria: $<10 \%$ - no expression, 11-40\% - low expression, 41-70\% - moderate expression, $>71 \%$ - high expression. SHED were examined for following markers: CD18, CD29, CD31, CD34, CD44, CD45, CD49d, CD49e, CD63, CD71, CD73, CD90, CD105, CD106, CD117, CD133, CD146, CD166, CD184, CD197, HLA I and HLA II.

\section{Results}

We isolated three stable lines from SHED (Figs. 1, 2). After 24 hours of cultivation, we first observed SHED as single cells or as small colonies (Fig. 3); non-adherent cells and the remnants of pulp tissue were washed out using PBS (Fig. 4). In primary cultures, after 5 days in vitro, we found colonies consisting of about 50 cells. Cells were ready for first passage at that time (Fig. 5). Subsequent passages were 
carried out after reaching $70 \%$ confluence (after $98.7 \mathrm{hrs}$ in average).

Compared to DPSC, SHED generated more rounded cells without long processes (Figs. 6, 7). Cumulated population doublings (PD) documented SHED proliferated over 45 PD (Graph 1). The average doubling time (DT) during long term cultivation was $41.3 \mathrm{hrs}(21.3-97.3 \mathrm{hrs})$. Initial DT for first 24 PD was 28.4 (21.3-34.6) hours. After reaching 24 PD, the doubling time had increased to 54.12 (32.7-97.3) hours (Graph 2). Average viability during long-term cultivation of SHED was $90.6 \%$ (86.0-94.0 \%). The diameter distribution of cells generated in culture showed a stable pattern during cultivation (12.24 -16.43 $\mu \mathrm{m}$ in diameter) with an average diameter of $15.0 \mu \mathrm{m}$. For the first $24 \mathrm{PD}$, the average cell diameter was $15.29(14.6-15.8) \mu \mathrm{m}$. After $24 \mathrm{PD}$, the average diameter was 14.66 (12.24-16.43) $\mu \mathrm{m}$ (Graph 3). During long term cultivation we did not observe any signs of culture degeneration or spontaneous differentiation and SHED were cytogenetically stable.

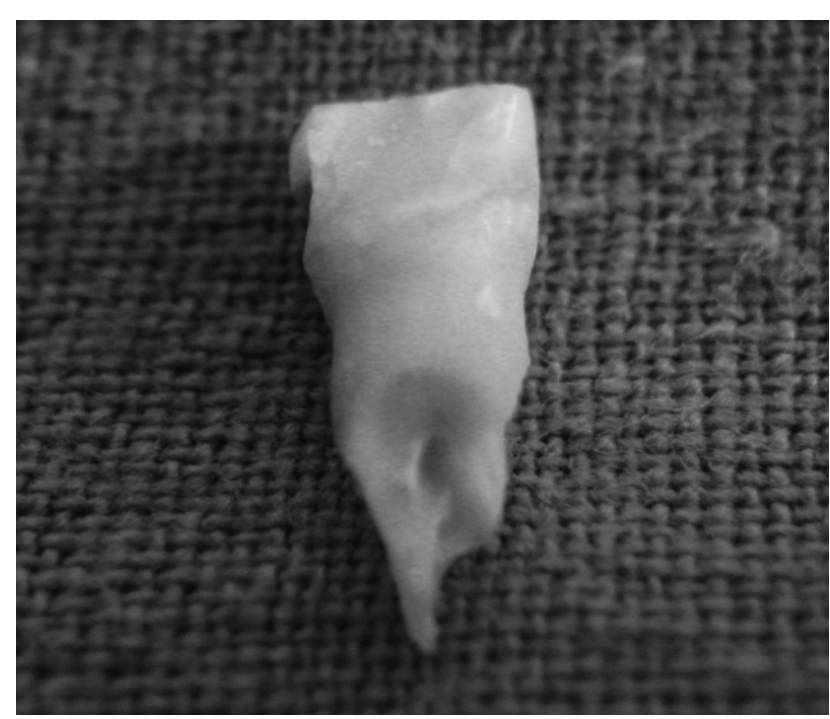

Fig. 1: Exfoliated deciduous middle incisor, with started resorption of the root and opened pulp chamber.

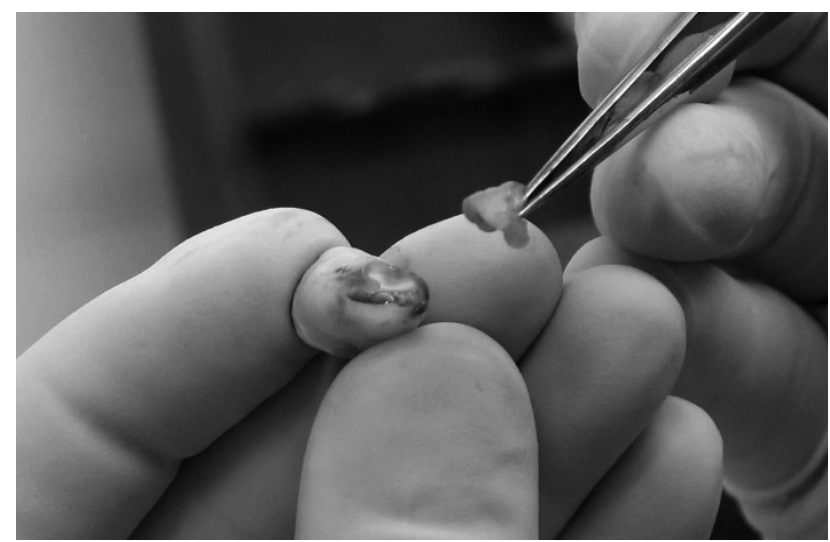

Fig. 2: Extracted dental pulp tissue.

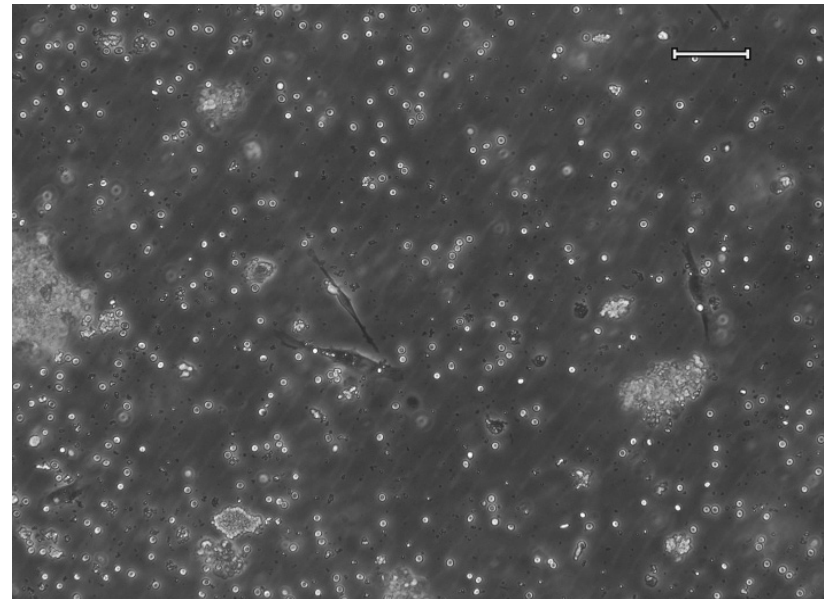

Fig. 3: Inoculated SHED (day 1) with remnants of the dental pulp. Phase contrast microscopy, magnification 200x.

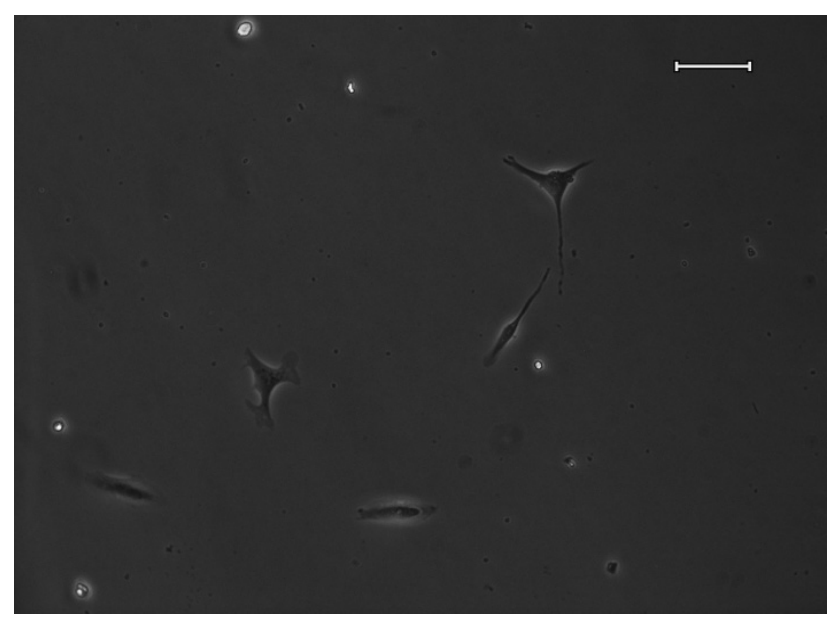

Fig. 4: Single SHED 24 hours following inoculation. Phase contrast microscopy, magnification 200x.

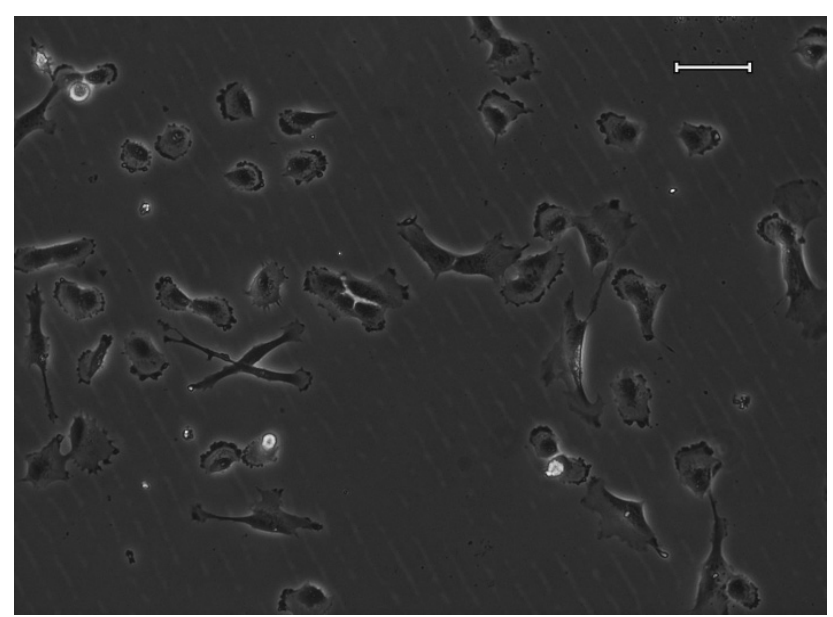

Fig. 5: Colony of SHED 5 days following inoculation. Primary culture is ready for first passaging. Phase contrast microscopy, magnification 200x. 


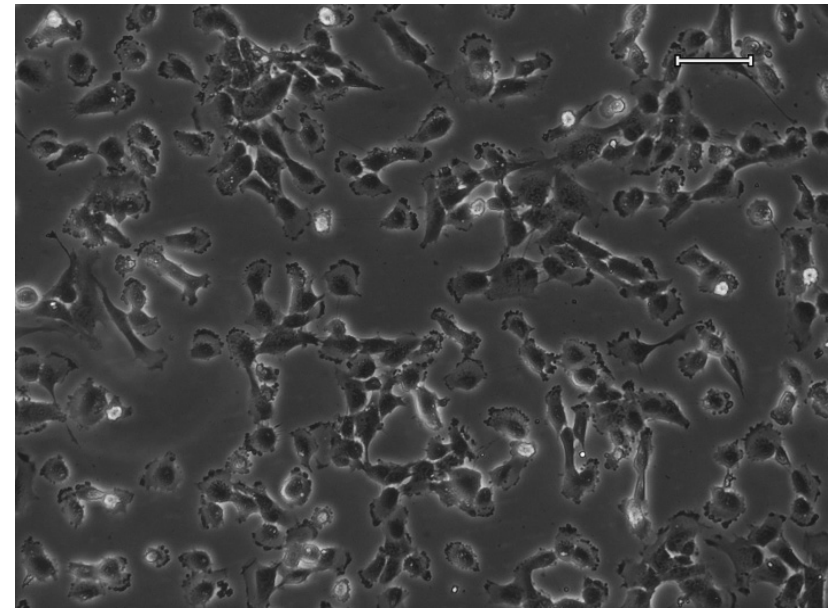

Fig. 6: SHED within first passage in $70 \%$ confluency. Compare to DPSC (Fig. 7) SHED are more rounded without long processes. Phase contrast microscopy, magnification 200x.

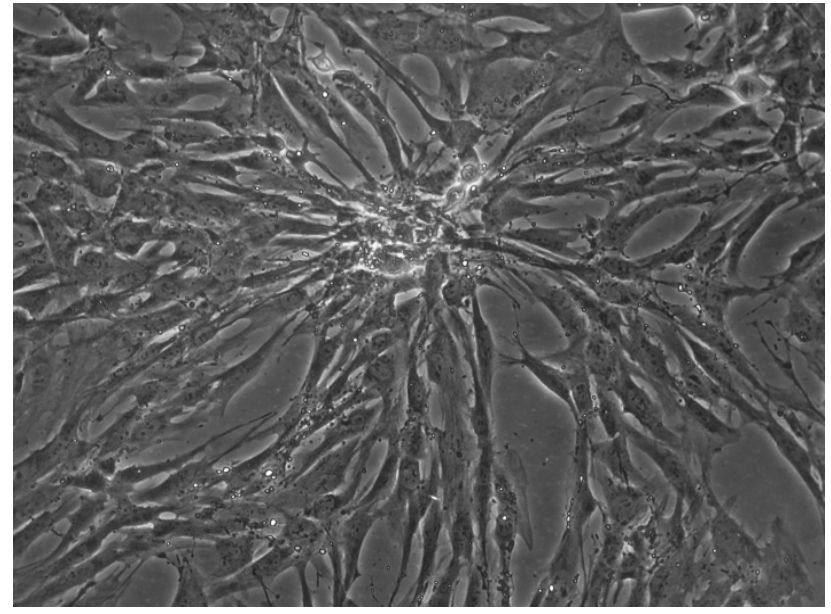

Fig. 7: DPSC in first passage in $70 \%$ confluency. Phase contrast microscopy, magnification 200x.

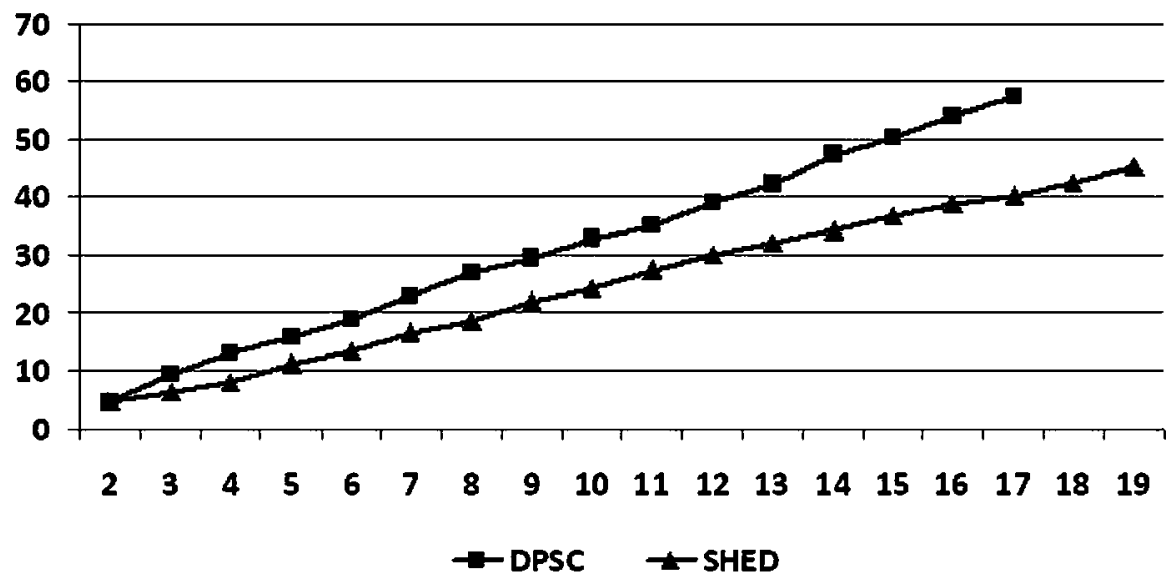

Graph 1: From $2^{\text {nd }}$ till $19^{\text {th }}$ passage, we were able to reach 45 PD with SHED. Compared to DPSC, where we reached 58 PD till $17^{\text {th }}$ passage.

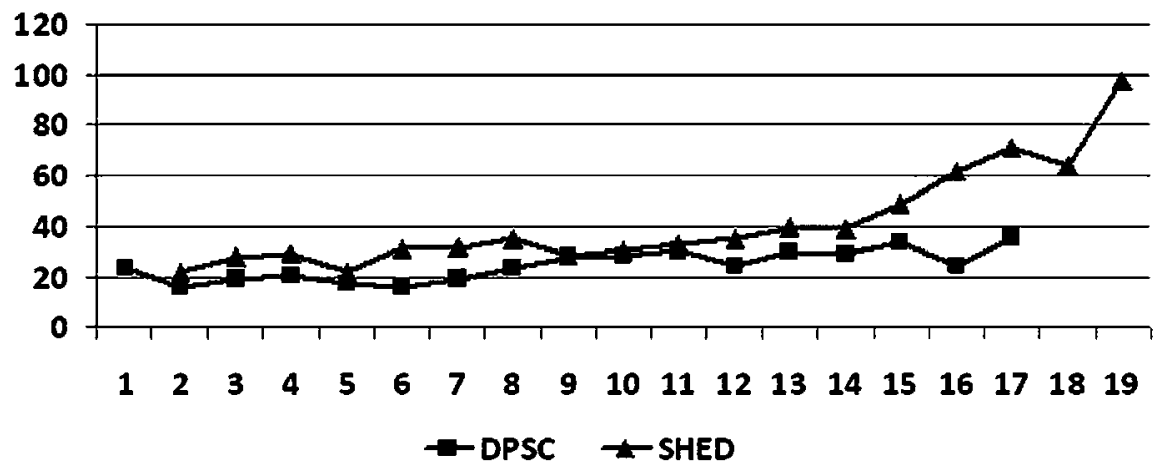

Graph 2: Doubling time trend during long term cultivation. SHED had higher average DT than DPSC. Average SHED DT was $60.8 \mathrm{hrs}$ (21.3-97.3 hrs), compared to DPSC $24.5 \mathrm{hrs}$ (15.55-35.12 hrs). For the first 24 PD SHED DT (28.4 hours) was about 33 \% higher than DT of DPSC (19.3 hours). After reaching 24 PD DT of SHED increased to 54.2 hours, while PD of DPSC increased only to 29.1 hours. 


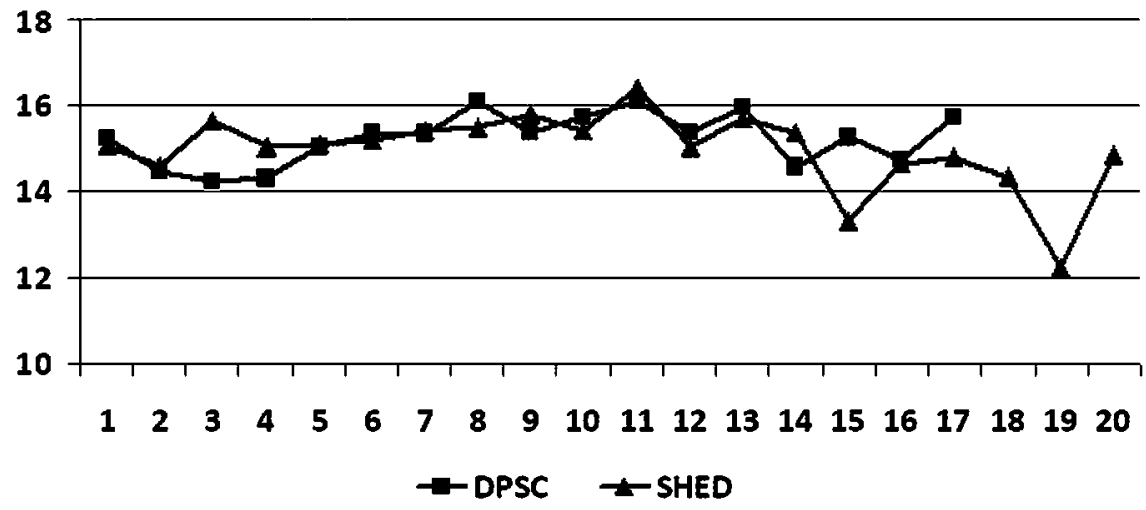

Graph 3: Diameter distribution of SHED compared to DPSC. SHED diameter distribution presented quite stable pattern during cultivation (12.24-16.43 $\mu \mathrm{m}$ in diameter) with average diameter of $15.0 \mu \mathrm{m}$.

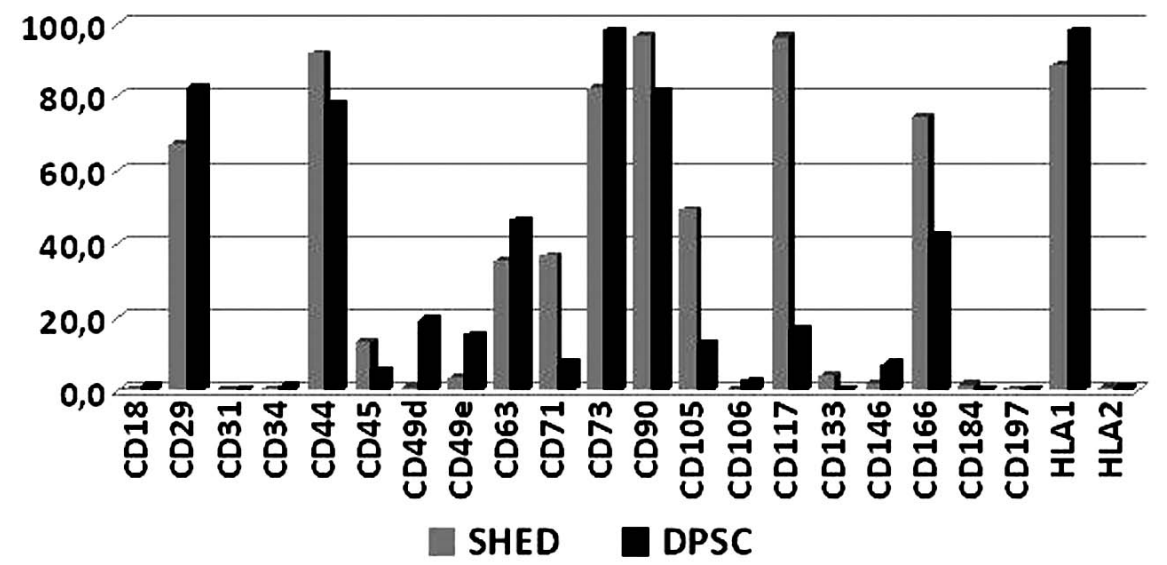

Graph 4: Compared to DPSC, SHED were significantly more positive for CD 71, CD 105, CD 117 and CD 166 and there were no significant changes in any other markers in our tested panel.

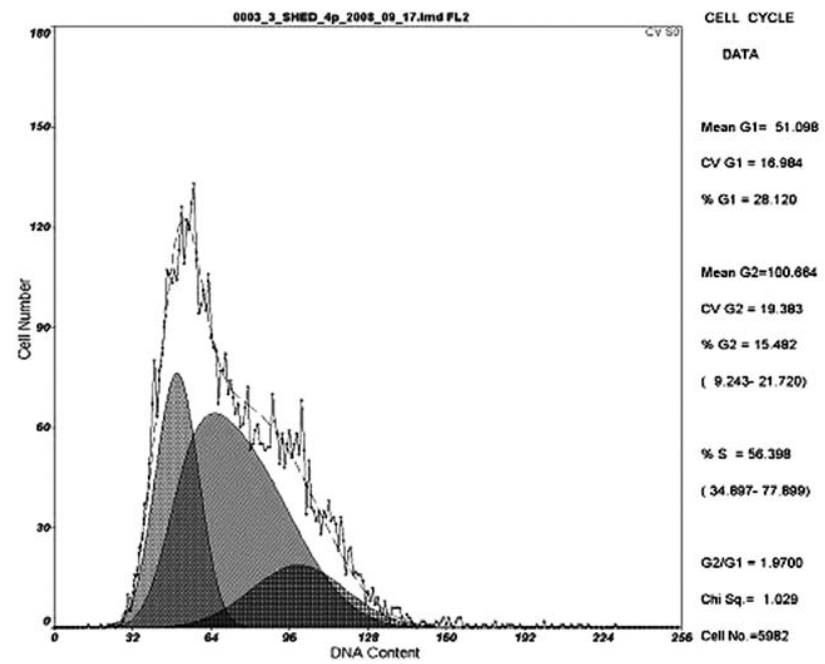

Graph 5: DNA analysis of SHED. Over $71 \%$ of cells is in $\mathrm{S}-\mathrm{G}_{2}$ phase.

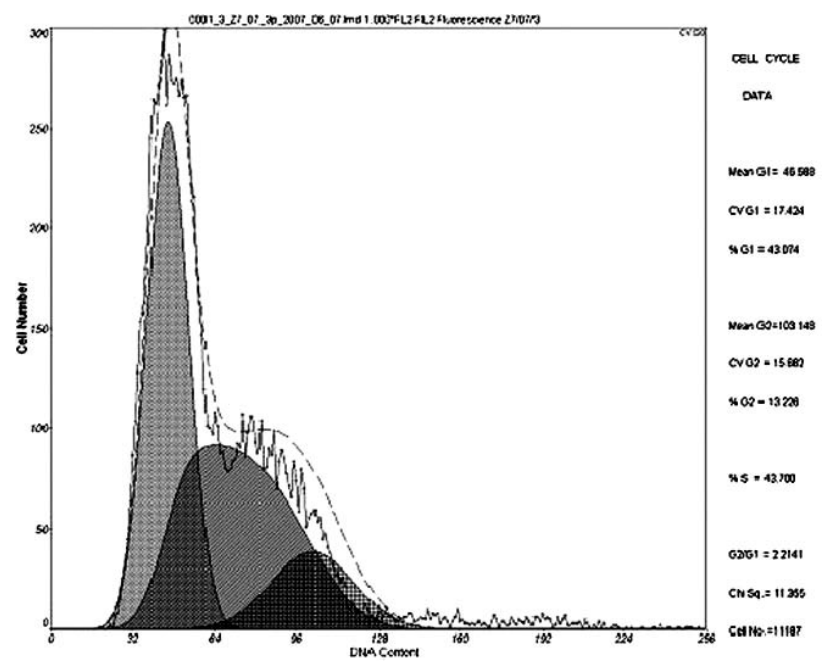

Graph 6: DNA analysis of DPSC. Over $58 \%$ of cells is in $\mathrm{S}-\mathrm{G}_{2}$ phase. 
Our phenotypic analyses of SHED showed high positivity for CD44 (91.7\%), CD73 (82.3\%), CD90 (96.5\%), CD117 (96.2 \%), CD166 (74.3 \%) and HLA I (88.3\%). Moderate positivity was observed for CD29 (67.0\%), CD105 (49.1\%) and low positivity was seen for CD45 (13.2\%), CD63 (35.1\%) and CD71 (36.6 \%). SHED progeny were negative for CD18 (0.1\%), CD31 (0.0\%), CD34 (0.1\%), CD49d (0.5\%), CD49e (3.5\%), CD106 (0.0\%), CD133 (3.9\%), CD146 (1.7\%), CD184 (1.3\%), CD197 (0.2\%) and HLA II (0.4\%) (Graph 4).

Propidium iodide-based DNA analysis showed regularly $70 \%$ of SHED being in $\mathrm{S}_{2} \mathrm{G}_{2}$ phase of cell cycle (Graph 5). Compare to DPSC where only about $57 \%$ of cells were in $\mathrm{S}-\mathrm{G}_{2}$ phase (Graph 6).

\section{Discussion}

The possibility of using stem cells, biological molecules and tissue engineering in clinical dentistry, opens completely new approaches to restore functionally and an aesthetically suitable tooth arch. For these purposes, dental pulp represents a source of stem cells which is well-delimited from other tissues. Furthermore exfoliated teeth or teeth extracted due to other reason (orthodontics, etc.) are uselessly discarded. In our previous study we investigated biological properties and basic phenotypic characteristics of DPSC $(8,9)$; in the present study, we focused on stem cells isolated from exfoliated teeth to determine if these stem cell lines possess similar (or different) properties. In our previous study, we cultivated SHED in medium which we modified for DPSC (9). With SHED line we were able to reach $45 \mathrm{PD}$, counted from the $2^{\text {nd }}$ passage. In primary cultures the amount of cells was very low; therefore we decided not to count SHED within the first passage to enhance the yield and number of cells seeded. For DPSC we obtained average 50 cells (10-110) after enzymatic dissociation, so we estimate that from primary culture to the counting in the first passage there were at least 10 PD. In comparison to DPSC, SHED had higher average DT. The average SHED DT was $41.3 \mathrm{hrs}$ (21.3-97.3 hrs) compared to DPSC 24.5 hrs (15.55-35.12 hrs). For the first 24 PD, SHED DT (28.4 hours) was about $33 \%$ higher than DT of DPSC (19.3 hours). After reaching 24 PD DT of SHED DT increased to 54.2 hours, while PD of DPSC increased only to 29.1 hours. The average diameter of both lines of our tested stem cell lines was comparable (SHED $15.0 \mu \mathrm{m}$ and DPSC $15.2 \mu \mathrm{m})$. But the diameter distribution of SHED varied in a wider range $(12.24-16.43 \mu \mathrm{m})$ compared that of DPSC (14.25-16.13 $\mu \mathrm{m})$.

Phenotypical analyses showed that, comparing to DPSC (CD $71-7.1 \%$, CD $105-12.6 \%$, CD $117-16.4 \%$ and CD $166-41.7 \%$ ) SHED had significantly (more than $20 \%$ of positive cells) higher positivity for CD 71, CD 105, CD 117 and CD 166 and there were no significant changes in any other markers in our tested panel. From these differences, we propose that SHED are more undifferentiated (high po- sitivity for CD 117 - receptor for stem cell factor I, typical for pluripotent cells) than DPSC and there should be better response for differentiation (CD 105 - a component of the TGF receptor). CD 146 function is still poorly understood, but it is proposed that CD 146 is a part of the endothelial junction associated with the actin cytoskeleton. Miura at al (5) found SHED positive for CD 146 whereas our lines were CD 146 negative. However, Miura et al. did not specify precisely how many CD 146 positive cells they found. Due to different cultivation media used, it is not presently possible to satisfactorily explain this difference. The cultivated cells are not hematopoietic, because they did not express CD 45. This is consistent with the negative expression of CD 34 and CD 18, which are another markers typical for hematopoietic stem cells. Moreover, they did not express CD 184 (SDF-1 receptor) which is by many authors associated with homing to bone marrow. On the other hand, SHED express medium positivity for CD 105, a marker which is commonly expressed by endothelial progenitors. Most reports showed co-expression of CD 105 and CD 133. We cannot explain why this co-expression was not observed, but this finding can support our idea- that SHED are unique undiferentiated stem cell lineage. The fact that SHED are undifferentiated is supported by no expresion of CD 31 (PECAM) and CD 106 (markers of endothelial differetiation). To our knowledge, no other evidence describing CD markers on SHED has yet been published.

The most surprising finding was slow proliferation of SHED compared to DPSC. DNA analysis showed $69.8 \%$ SHED to be in $\mathrm{S}_{-} \mathrm{G}_{2}$, but in DPSC lines, only $56 \%$ cells resided in the $\mathrm{S}_{-} \mathrm{G}_{2}$ phase. Moreover, SHED displayed an increased positivity for CD 71, a marker found on proliferating cells. In addition, SHED showed increased surface expression of CD 105 also known as endoglin, an endothelial cell marker. These data are consistent with the conclusion that SHED hold a higher proliferation potential than DPSC lines. However, our cultivation medium was optimized for DPSC and may not be fully suitable for SHED. In future experiments we will optimize cultivation media for SHED to provide a firmer foundation for our interpretation of these phenotypic differences.

\section{Conclusions}

We isolated and expanded ex vivo stem cells from exfoliated teeth and cultivated them over Hayflick's limit. Cultivated SHED were highly proliferative and cytogenetically stable stem cells. Over the entire cultivation period, we did not observe changes in cell viability or differentiation; the cells remained undifferentiated. Dental pulp of exfoliated teeth thereby represents alternative and easily accessible source of tissue-specific stem cells which are histocompatible with patient -specific tissues. SHED were stable after cryopreservation and can be therefore be quite useful for stem cell tissue banks. Dental pulp tissue from exfoliated deciduous teeth represents an easily accessible 
source of tissue; one which is often discarded, but which instead may be useful as source of stem cells for research and clinical applications.

\section{Acknowledgments}

Work was supported by grant project of the Ministry of Health, Czech Republic NR 9182-3/07.

\section{References}

1. Chai Y, Slavkin HC. Prospects for tooth regeneration in the $21^{\text {st }}$ century: A perspective. Microscopy research and technique. 2003; 60: 469-479

2. Gotlieb E. L, Murray P. E, Namerow K. N, Kuttler S, Garcia-Godoy F. An ultrastructural investigation of tissue-engineered pulp constructs implanted within endodontically treated teeth. J Am Dent Assoc 2008; 139: 457-465.

3. Gronthos S, Cherman N, Robey P, Shi S. Human dental pulp stem cells. Adult Stem Cells. Totowa, New Jersey: Humana Press, 2004:37-51,101-149.
4. Gronthos S, Mankani M, Brahim J, et al. Postnatal human dental pulp stem cells (DPSCs) in vitro and in vivo. Proc Natl Acad Sci USA 2000;97:13625-13630.

5. Miura M, Gronthos S, Zhao M, Lu B, Fisher LW, Robey PG, Shi S. SHED: Stem cells from human exfoliated deciduous teeth. Proc Natl Acad Sci USA 2003; 100:5807-5812

6. Pereira, R.F., Halford, K.W., O'Hara, M.D., et al.: Cultured adherent cells from marrow can serve as long-lasting precursor cells for bone, cartilage and lung in irradiated mice. Proc Natl Acad Sci USA, 1995, č. 92, s. 4857-61.

7. Sanchez-Ramos, J. et al.: Adult bone marrow stromal cells differentiate into neural cells in vitro. Exp Neurol, 2000, č. 164, s. 247-256.

8. Suchánek J, Soukup T, Ivančaková R, et al. Human dental pulp stem cell - isolation and long term cultivation. Acta Med (Hradec Kralove) 2007; 50: 195-201

9. Suchánek J., Soukup T., Višek B., et al. Dental pulp stem cells and their characterization. Biomed Pap Med Fac Univ Palacky Olomouc Czech Rep. 2009, 153(1):1-6. 10. Turksen K. Adult Stem Cells. Totowa, New Jersey: Humana Press, 2004:37-51, 101-149.

11. Yen, AHH; Sharpe, PT. Regeneration of teeth using stem cell-based tissue engineering. Expert opinion on biological therapy. 2006; 6: 9-16.

12. Yu J, Deng Z, Shi J, Zhai H, Nie X, Zhuang H, Li Y, Jin Y. Differentiation of dental pulp cells into regular-shaped dentin pulp complex induced by tooth germ cell conditioned medium. Tissue Eng. 2006; 11:3097-3105.

Received: 08/02/2010.

Accepted in revised form: 06/05/2010.

\section{Corresponding author:}

Jakub Suchánek, Department of Dentistry, Charles University in Prague, Faculty of Medicine in Hradec Králové, Sokolská 581, 50005 Hradec Králové, Czech Republic; e-mail: suchanekj@lfhk.cuni.cz 